\title{
Patterns of Jaw Coarticulatory Direction and Linguomandibular Coordination in VCV Sequences
}

\author{
Daniel Recasens \\ Institut d'Estudis Catalans and \\ Universitat Autònoma de Barcelona
}

\section{$1 \quad$ Introduction}

Data on lingual movement, dorsopalatal contact and F2 frequency presented in previous papers of ours (Recasens, 2002; Recasens and Pallarès, 2001; Recasens, Pallarès and Fontdevila, 1997) suggest that the degree of articulatory constraint (DAC) model accounts to a large extent for the extent and direction of tongue dorsum coarticulation in VCV and CC sequences. A goal of this investigation is to verify the predictions of this model with respect to jaw V-to-V effects in VCV sequences using articulatory movement data collected with electromagnetic articulometry (EMA).

\subsection{The DAC model}

According to the DAC model of coarticulation, consonants may be more or less constrained based on demands on place and manner of articulation. Some consonants appear to be specified for a high degree of articulatory constraint, i.e., dorsals (in agreement with the sluggishness of the tongue dorsum and the large contact area involved in their production), lingual fricatives and trills (in line with the precise mechanisms used by speakers for their implementation, namely, tongue grooving for the former and adequate tongue shape and elasticity for the latter), and dark /1/ (since this realization requires the formation of a secondary postdorsal constriction at the upper pharynx). Regarding consonants specified for a lower degree of tongue dorsum constraint, coupling between the tongue dorsum and the primary tongue front articulator causes dentals and alveolars to be more constrained than bilabials. Differences in tongue predorsum activation render /i/ more constrained than /a/ and $/ \mathrm{u} /$, and $/ \mathrm{\partial} /$ more unconstrained than the other vowels.

The degree of constraint for the intervocalic consonant has important consequences for the extent and direction of the coarticulatory effects in VCV sequences. Indeed, the DAC value for the consonant is inversely related to the degree of $\mathrm{V}$-to-C coarticulation (i.e., to how sensitive the consonant is to the influence of the adjacent vowels) and positively related to the $\mathrm{C}$-to-V effects (i.e., to how much the consonant affects the adjacent vowels). Moreover, as shown below, requirements on consonantal production may also explain whether C-to-V anticipation prevails over $\mathrm{C}$-to- $\mathrm{V}$ carryover, or viceversa. Trends in $\mathrm{V}$-to- $\mathrm{V}$ direction may also be accounted for assuming that vocalic anticipation ought to vary inversely with the prominence of the consonantal carryover effects while vocalic carryover effects are expected to decrease with the strength of the consonant-dependent anticipatory component. The following patterns of C-to- $\mathrm{V}$ and $\mathrm{V}$-to- $\mathrm{V}$ coarticulatory direction may be identified:

(a) Among consonants with a high DAC value, dark /1/ favors C-to-V1 anticipation over C-to-V2 carryover consistently with the fact that tongue dorsum lowering and retraction often starts before tongue tip raising for the implementation of this consonantal realization. On the other hand, consonants involving tongue dorsum raising such as alveolopalatals and 
velars favor C-to-V2 carryover effects in accordance with the slow motion of the primary dorsal articulator at consonantal release which may be due to inertia.

In agreement with these C-to- $\mathrm{V}$ patterns, data reported in our previous papers reveal that VCV sequences with dark /l/ allow more vocalic anticipation than vocalic carryover while those with the alveolopalatal /n/ show more vocalic carryover than vocalic anticipation. A specific situation applies to VCV sequences with dorsovelars: vocalic effects from /i/ vs /a/ favor the expected carryover direction when the transconsonantal vowel is /i/ (e.g., in the sequence pair /aki/-/iki/) but the anticipatory direction when the transconsonantal vowel is /a/ presumably since forward tongue dorsum motion during the velar closure period causes extensive vocalic anticipation to occur (e.g., in the sequence pair /aki/-/aka/).

(b) Two basic patterns of C-to-V direction are found in VCV sequences with dentals and alveolars but for dark /1/ (and, to some extent, for labials as well). Consonantal effects on /a/ happen to be more prominent at the anticipatory level presumably since this vowel permits free apical anticipation; on the other hand, C-to-V effects on /i/ are especially salient at the carryover level since this vowel contributes to tongue dorsum raising during the consonant. Accordingly, vowel effects appear to favor the carryover component in the latter scenario (e.g., in the sequence pair /ati/-/iti/) and the anticipatory direction in the former one (e.g., in the pair /ati/-/ata/).

\subsection{Jaw coarticulation}

Data on jaw coarticulation are scarce. To a large extent, trends in C-to-V coarticulation for the jaw resemble those for the tongue dorsum in the light of evidence showing that the former articulatory structure may assist the latter along the height dimension (e.g., high vowels involve both tongue body and jaw raising while low vowels are produced with a lowered tongue body and jaw). Indeed, previous studies addressing this issue (Keating, Lindblom, Lubker and Kreiman, 1994) reveal that high vowels are more resistant than low vowels to Cto- $\mathrm{V}$ effects in jaw height, and that such effects become more prominent as a function of consonants involving a higher jaw (dentoalveolars including /s/ but not so /l/) than of those produced with a lower jaw (labials, /l/, velars). Compatibly, consonants of the latter group are more likely to be influenced by $\mathrm{V}$-to-C effects in jaw height than those of the former group.

Regarding coarticulatory direction, data in the literature reveal that the jaw often favors the carryover over the anticipatory component both for the consonantal and for the vocalic effects which could be associated with the relatively slow motion of the mandible in speech. This trend is documented in VCV sequences with labial consonants, both in the light of articulatory data (Tuller, Harris and Gross, 1981; Sussman, MacNeilage and Hanson, 1973) and of acoustic data on F1 coarticulatory effects from /i/ vs /a/ in English /VbəbV/ sequences (Magen, 1997). (F1 is known to reflect changes in mouth opening degree and should thus be correlated with variations in jaw height). In a recent F1 coarticulation study with the same consonants included in the present investigation (Recasens and Pallarès, 2000), C-to-V and Vto- $\mathrm{V}$ effects from /i/ vs /a/ in VCV sequences with transconsonantal /a/ (e.g., /iCa/-/aCa/) were also found to be more prominent at the carryover level than at the anticipatory level; on the other hand, V-to-V effects in sequences with transconsonantal /i/ (e.g., /iCa/-/iCi/) were reported to favor the anticipatory direction in sequences with lingual fricatives, and the carryover direction in those with consonants produced with a low jaw (dark /l/, velars). Other studies report however considerable anticipatory and carryover vocalic effects in jaw coarticulation in VCV sequences with bilabial, alveolar and velar stops (Fletcher and Harrington, 1999). 


\section{$2 \quad$ Method}

\subsection{Recording procedure}

Three Catalan speakers uttered ten times all possible VCV combinations with the consonants $/ \mathrm{p}, \mathrm{n}, \mathrm{l}, \mathrm{s}, \int, \mathrm{n}, \mathrm{k} /$ (where $/ \mathrm{l} /$ is dark and $/ \mathrm{n} /$ is an alveolopalatal) and the vowels /i, a, u/ embedded in the Catalan sentence ['graßə pV'CVp ə'ßans] ("He records pVCVp earlier"). The inclusion of contextual labial consonants and a schwa ensured that the vowel-dependent coarticulatory effects of interest could be expanded sufficiently along the time domain.

Articulatory movement and acoustic data were collected simultaneously using electromagnetic articulometry by means of a Carstens Articulograph system AG-100. This system consists of a head mount with three magnetic transmitters that generate a magnetic field, and a set of small transducer coils that can be attached to different articulatory structures in the midsagittal plane. As the articulators move inside the vocal tract, the transducer coils induce a signal that is inversely proportional to the cube of the distance between transmitter and transducer. The resulting signal results in a set of voltages which can be converted to distance. In the present experiment coils were placed on the tongue tip, tongue blade, tongue dorsum, incisors of the lower jaw, and upper and lower lip, as well as on the bridge of the nose and upper incisors for head movement correction. Estimates for the subjects' occlusal planes were obtained as anatomical references to which the data could be rotated, as well as traces of their palates.

Movement and acoustic data were digitized using a real-time input system at a sampling rate of $250 \mathrm{~Hz}$ for movement and $10 \mathrm{kHz}$ for speech; the time resolution of the EMA data was 4 ms. The kinematic data were converted from voltage to distance, corrected for head movement, rotated to the occlusal plane, and extracted into separate articulatory channels for the $\mathrm{X}$ and $\mathrm{Y}$ dimensions.

\subsection{Data analysis}

For each VCV repetition, the onset and offset of the intervocalic consonant were identified from spectrographic and waveform displays, and occasionally from the movement data according to criteria summarized elsewhere (Recasens, 2002). The temporal extent of V-to-V coarticulatory effects was analyzed from the onset of $[\beta]_{1}$ to the offset of $[\beta]_{2}$ in the sequence $[\beta ə \# \# p V C V p \# \# ə \beta]$.

Vocalic temporal effects were considered to occur as long as a significant voweldependent difference in articulatory displacement (i.e., /i/ vs /a/, /i/ vs /u/ and /a/ vs /u/, referred to as 'changing' vowel in this paper) extends into the consonant and the transconsonantal vowel (i.e., /i/, /a/ and /u/, referred to as 'fixed' vowel from here forwards). Significant differences as a function of changing vowel for each consonant and fixed vowel condition were computed for X and Y movement data for all six articulatory regions TT, TL, TD, J, UL and LL: results for TDX, TDY and TTX were presented in Recasens (2002) and those for JY will be given in the present paper. V-to-V mandibular effects were measured in jaw height only (i.e., for changing /i/ vs /a/ and /a/ vs /u/) considering the close relationship between jaw and tongue dorsum elevation and the fact that jaw horizontal movement is often small in speech production.

In order to carry out the statistical evaluation of interest, mean articulatory trajectories across repetitions for a given asymmetrical sequence (e.g., effects from $\mathrm{V} 2=/ \mathrm{i} / \mathrm{on} / \mathrm{h} /$ and $\mathrm{V} 1=/ \mathrm{a} /$ ) were compared with those in symmetrical sequences composed of the same consonant and the same fixed transconsonantal vowel (i.e., /ana/). One-way ANOVAs 
Scheffé $(p<0.05)$ were applied every 4 ms starting at consonantal offset back to $[\beta]_{1}$ onset in order to determine the extent of vocalic anticipation and from consonantal onset until $[\beta]_{2}$ offset in order to estimate the extent of vocalic carryover. ([$[\beta]_{1}$ onset and $[\beta]_{2}$ offset were identified with the shortest temporal values for a given pair of symmetrical and asymmetrical VCV sequences across repetitions). The last significant difference counting backwards during fixed V1 was taken to be the onset of a V2-dependent anticipatory effect and the last significant difference counting forwards during fixed V2 was taken to be the offset of a V1dependent carryover effect.

Significant coarticulation times obtained according to the procedure just described are shown in Table I across speakers. Those values were submitted to further statistical analysis in view of the large speaker-dependent variability involved (see standard deviations in the table). Repeated measures ANOVAs and post-hoc tests (Scheffé) were performed with speaker as a factor and coarticulation time as the dependent variable $(\mathrm{p}<0.05)$. Two analyses were carried out. In test 1, main effects and interactions for JY were computed for the independent variables 'direction' (anticipatory, carryover), 'changing vowel' (/i/ vs /a/, /a/ vs $/ \mathrm{u} /$ ) and 'consonant' (/p, n, l, s, $, \mathrm{n}, \mathrm{k} /)$. In order to evaluate the role of the fixed vowel in the duration of the coarticulatory effects, test 2 was performed for the variables 'direction', 'consonant' and 'fixed vowel' separately for changing front /i/ vs back /a/ (test 2a) and for changing low back /a/ vs high back /u/ (test $2 \mathrm{~b}$ ). Significant effects for the mean values of interest are presented in Table II.

\section{$3 \quad$ Results}

\subsection{Coarticulatory durations}

(a) JY data yielded no significant main effects but two significant interactions according to results from test 1 , i.e., changing vowel $x$ direction $(F(2,2)=4.108, p<0.052)$ and consonant $x$ direction $(\mathrm{F}(2,12)=4.227, \mathrm{p}<0.004)$. The former interaction is associated with differences in carryover duration for /i/ vs /a/ $(189 \mathrm{~ms})>/ \mathrm{a} / \mathrm{vs} / \mathrm{u} /(127 \mathrm{~ms})$, and the latter interaction with longer effects for $/ \mathrm{n} /$ than for $/ \mathrm{s} /$ also at the carryover level. Coarticulatory durations in the consonant $\mathrm{x}$ direction panels of Table II reveal indeed that, in comparison to the other consonants, those for $/ \mathrm{n} /$ are longer at the carryover level while those for $/ \mathrm{s} /$ are shorter at the carryover level and longer at the anticipatory level.

These coarticulation trends are similar to those for TDY (correlation coefficients between the coarticulatory durations for JY and for TDY yielded an overall $r$ value of 0.57 ). Indeed, a comparison between the dotted and continuous lines at the right bottom graph of Figure 1 reveals similar V-to-V coarticulatory durations both for TDY and JY in the case of VCV sequences with dorsal $/ \mathrm{n} /$ and, less so, in those with $/ \mathrm{k} /$ (correlation coefficients were 0.92 for the former sequences and 0.74 for the latter). Moreover, a more detailed inspection of the $\mathrm{V}$-to- $\mathrm{V}$ coarticulatory durations for both dorsal consonants reveals that they are longer in sequences with /i/ than in those without/i/. Vocalic effects for the fricatives yielded lower correlation values between TDY and JY ( $r$ values were 0.74 for $/ \mathrm{s} /$ and 0.52 for $/ / /$ ) and, as shown by the left bottom graph in the figure, those in sequences without /i/ were often longer than those in sequences with /i/. Correlation values were also high for $/ \mathrm{p} /(0.80)$ but not so for the alveolars /n/ and /1/ (see top graph of Figure 1). 
Table I. Temporal extent of significant V-to-V anticipatory and carryover effects for JY across speakers. Data (in ms) are listed as a function of consonant, and changing and fixed vowel condition. Standard deviations are also given in italics.

\begin{tabular}{|c|c|c|c|c|}
\hline & \multicolumn{2}{|c|}{ Changing $\underline{\mathbf{i}}$ vs $\underline{a}$} & \multicolumn{2}{|c|}{ Changing a vs $\underline{\mathrm{u}}$} \\
\hline & (fixed $\underline{i})$ & (fixed $\underline{a})$ & (fixed $\underline{a})$ & (fixed $\underline{u})$ \\
\hline \multirow[t]{2}{*}{$\mathbf{p}(A n t)$} & 168 & 169 & 256 & 64 \\
\hline & 156 & 96 & 118 & 46 \\
\hline \multirow[t]{2}{*}{$\mathbf{p}(\mathrm{Car})$} & 139 & 245 & 164 & 35 \\
\hline & 140 & 175 & 80 & 33 \\
\hline \multirow[t]{2}{*}{$\mathbf{n}(A n t)$} & 173 & 137 & 151 & 127 \\
\hline & 150 & 103 & 73 & 134 \\
\hline \multirow[t]{2}{*}{$\mathbf{n}(\mathrm{Car})$} & 240 & 228 & 132 & 112 \\
\hline & 170 & 139 & 136 & 161 \\
\hline \multirow[t]{2}{*}{$\mathbf{l}(A n t)$} & 185 & 137 & 173 & 175 \\
\hline & 145 & 130 & 141 & 147 \\
\hline \multirow[t]{2}{*}{$\mathbf{l}($ Car $)$} & 191 & 131 & 121 & 100 \\
\hline & 149 & 169 & 104 & 52 \\
\hline \multirow[t]{2}{*}{$\mathbf{s}(A n t)$} & 220 & 199 & 167 & 368 \\
\hline & 173 & 155 & 176 & 11 \\
\hline \multirow[t]{2}{*}{$\mathbf{s}($ Car $)$} & 115 & 89 & 20 & 117 \\
\hline & 199 & 144 & 22 & 151 \\
\hline \multirow[t]{2}{*}{$\int(A n t)$} & 253 & 197 & 263 & 231 \\
\hline & 188 & 174 & 191 & 175 \\
\hline \multirow[t]{2}{*}{$\int(C a r)$} & 179 & 165 & 217 & 16 \\
\hline & 187 & 178 & 168 & 7 \\
\hline \multirow[t]{2}{*}{$\mathbf{j}(A n t)$} & 92 & 240 & 168 & 121 \\
\hline & 114 & 80 & 177 & 129 \\
\hline \multirow[t]{2}{*}{$\mathbf{n}(C a r)$} & 335 & 287 & 261 & 235 \\
\hline & 31 & 127 & 171 & 8 \\
\hline \multirow[t]{2}{*}{$\mathbf{k}(A n t)$} & 251 & 181 & 195 & 213 \\
\hline & 84 & 86 & 134 & 127 \\
\hline \multirow[t]{2}{*}{$\mathbf{k}($ Car $)$} & 131 & 173 & 172 & 75 \\
\hline & 145 & 178 & 118 & 91 \\
\hline
\end{tabular}


Table II. Mean values across speakers (in ms) and significant differences (in brackets) for JY vowel-to-vowel coarticulation times. Data for the consonant $\mathrm{x}$ direction condition are plotted as a function of changing vowel (columns) and consonant and direction (rows). Data for the consonant $\mathrm{x}$ fixed vowel condition are presented as a function of changing vowel (columns) and consonant and fixed vowel (rows).

\section{Consonant $x \underline{\text { direction }}$}

\begin{tabular}{|c|c|c|c|}
\hline & $\begin{array}{l}(\underline{\mathbf{i}} \text { vs } \underline{\mathbf{a}} ; \\
\underline{\mathbf{a}} \text { vs } \underline{\mathbf{u}})\end{array}$ & (i vs a) & $(\underline{\mathbf{a}}$ vs $\underline{\mathbf{u}})$ \\
\hline $\mathbf{p}(A n t)$ & 164 & 169 & 160 \\
\hline p (Car) & 146 & 192 & 99 \\
\hline $\mathbf{n}(A n t)$ & 147 & 155 & 139 \\
\hline n (Car) & 178 & 234 & 122 \\
\hline $\mathbf{l}(A n t)$ & 168 & 161 & 174 \\
\hline I (Car) & 136 & 161 & 111 \\
\hline $\mathbf{s}(A n t)$ & 238 & 209 & 267 \\
\hline s (Car) & 85 & 102 & 69 \\
\hline $\int(A n t)$ & 236 & 225 & 247 \\
\hline $\int$ (Car) & 144 & 172 & 117 \\
\hline $\mathbf{n}(A n t)$ & 155 & 166 & 145 \\
\hline $\mathbf{n}$ (Car) & 279 & 311 & 248 \\
\hline $\mathbf{k}(A n t)$ & 210 & 216 & 204 \\
\hline $\mathbf{k}$ (Car) & 138 & 152 & 123 \\
\hline
\end{tabular}

\section{$\underline{C} \times \underline{\text { fixed } V}$}

\begin{tabular}{|c|c|}
\hline & $(\underline{\mathbf{i}}$ vs $\underline{\mathbf{a}})$ \\
\hline $\mathbf{p}($ fixed $\underline{i})$ & 153 \\
\hline $\mathbf{p}($ fixed $\underline{a})$ & 207 \\
\hline $\mathbf{n}($ fixed $\underline{i})$ & 207 \\
\hline n (fixed $\underline{a})$ & 183 \\
\hline $\mathbf{I}($ fixed $\underline{i})$ & 188 \\
\hline $\mathbf{I}($ fixed $a)$ & 134 \\
\hline $\mathbf{S}$ (fixed $\underline{i})$ & 167 \\
\hline $\mathbf{s}($ fixed $a)$ & 144 \\
\hline $\int($ fixed $\underline{i})$ & 216 \\
\hline $\int($ fixed $a)$ & 181 \\
\hline $\mathbf{n}($ fixed $\underline{i})$ & 213 \\
\hline $\mathbf{n}($ fixed $a)$ & 263 \\
\hline $\mathbf{k}($ fixed $\underline{i})$ & 191 \\
\hline $\mathbf{k}($ fixed $a)$ & 177 \\
\hline
\end{tabular}

\begin{tabular}{|c|c|}
\hline & (a vs $\underline{\text { u}})$ \\
\hline $\mathbf{p}($ fixed $\underline{a})$ & 210 \\
\hline p (fixed $\underline{u})$ & 49 \\
\hline $\mathbf{n}($ fixed $\underline{a})$ & 141 \\
\hline n (fixed $\underline{u})$ & 119 \\
\hline $\mathbf{l}($ fixed $\underline{a})$ & 147 \\
\hline$I$ (fixed $\underline{u})$ & 137 \\
\hline $\mathbf{S}($ fixed $\underline{a})$ & 93 \\
\hline s $($ fixed $\underline{u})$ & 243 \\
\hline $\int($ fixed $\underline{a})$ & 240 \\
\hline $\int($ fixed $\underline{u})$ & 123 \\
\hline $\mathbf{n}($ fixed $\underline{a})$ & 215 \\
\hline $\mathbf{n}($ fixed $\underline{u})$ & 178 \\
\hline $\mathbf{k}($ fixed $\underline{a})$ & 183 \\
\hline $\mathbf{k}($ fixed $\underline{u})$ & 144 \\
\hline
\end{tabular}


(b) According to the two consonant $\mathrm{x}$ fixed panels in Table II, V-to-V coarticulatory effects associated with changing /a/ vs / $\mathrm{u} /$ were longer for fixed /a/ than for fixed $/ \mathrm{u} /$ for most consonants (right panel) while those associated with changing /i/ vs /a/ were generally longer when the fixed vowel was /i/ than when it was /a/ (left panel). Differently from these JY effects, V-to-V effects for TDY (see Recasens, 2002) were consistently longer in the fixed /a/ and $/ \mathrm{u} /$ condition than in the fixed /i/ condition.

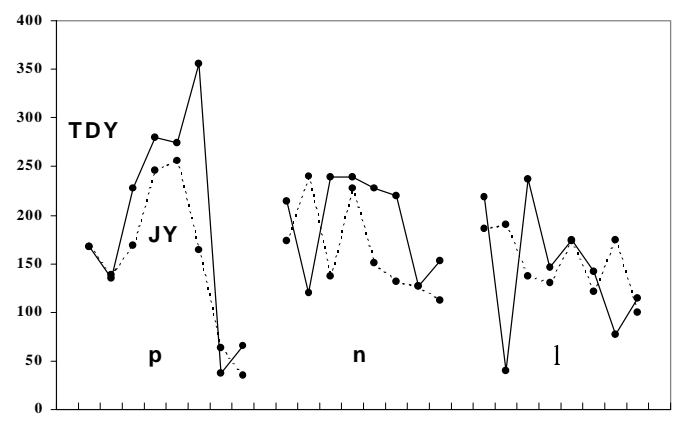

12345678
[1] i vs a (fixed i, anticipatory)

[2] i vs a (fixed i, carryover)

[3] i vs a (fixed a, anticipatory)

[4] i vs a (fixed a, carryover)

[5] a vs u (fixed a, anticipatory)

[6] a vs u (fixed a, carryover)

[7] a vs u (fixed u,anticipatory)

[8] a vs u (fixed u, carryover)
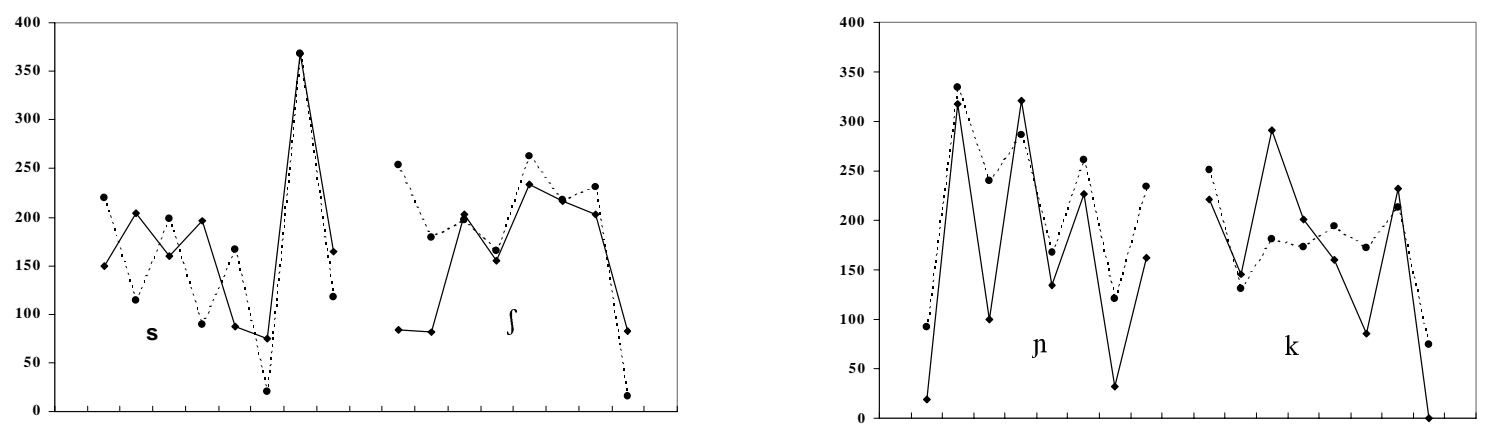

Figure 1. V-to-V coarticulatory durations for TDY and JY. Effects are displayed as a function of the intervocalic consonant in the changing and fixed vowel conditions.

\subsection{Coarticulatory direction}

Figure 2 displays differences in V-to-V coarticulatory direction for JY across speakers. Data on V-to-V coarticulatory direction for TDY are also given for comparison (see Recasens, 2002). In order to obtain those differences, the vowel-dependent carryover effects were subtracted from the vowel-dependent anticipatory effects for each consonant and all pairs of changing vowels in each fixed vowel context condition. Bars in the figure plot differences between carryover and anticipatory effects for changing /i/ vs /a/ in the fixed /i/ and /a/ conditions (dark bars), and for changing $/ \mathrm{a} / \mathrm{vs} / \mathrm{u} /$ in the fixed $/ \mathrm{a} / \mathrm{and} / \mathrm{u} /$ conditions (white bars).

The JY coarticulatory effects appear to favor the same direction as the TDY effects in VCV sequences with dorsal consonants $/ \mathrm{n} /$ and $/ \mathrm{k} /$, i.e., vocalic carryover for $/ \mathrm{n} /$ and vocalic anticipation for $/ \mathrm{k} /$. Regarding VCV sequences with the lingual fricatives $/ \mathrm{s} /$ and $/ \mathrm{J} / \mathrm{JY}$ effects are anticipatory rather than carryover, and the relative prominence of the anticipatory vs carryover effects is more obvious for JY than for TDY mostly so in the fixed vowel /i/ condition. 


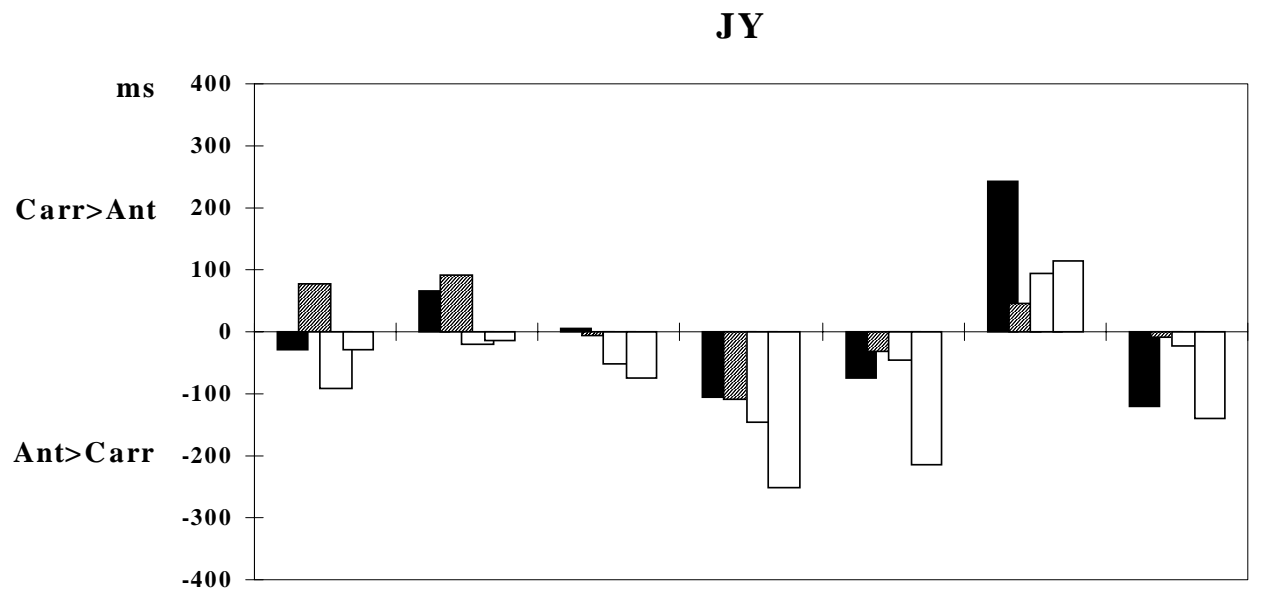

TDY

[1] i vs a (fixed i)

[2] i vs a (fixed a)

[3] a vs u (fixed a)

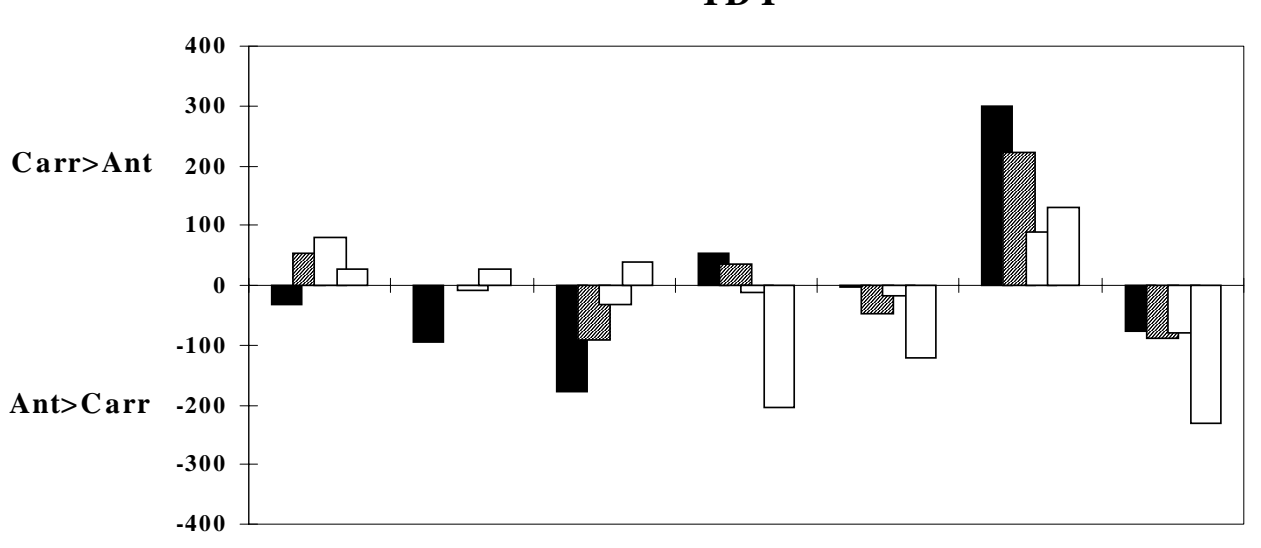

[4] a vs u (fixed $u$ )

1234

$\begin{array}{lllllll}\mathrm{p} & \mathrm{n} & \mathrm{l} & \mathrm{s} & \mathrm{j} & \mathrm{n} & \mathrm{k}\end{array}$

Figure 2. C-A differences in temporal extent of V-to-V coarticulation for JY (top) and TDY (bottom) across speakers. Data are displayed for different consonants and changing and fixed vowels. Positive values indicate prevalence of the carryover over the anticipatory direction, and negative values the opposite relationship.

Jaw and tongue dorsum coarticulation data for $/ \mathrm{p} /, / \mathrm{n} /$ and dark $/ \mathrm{l} / \mathrm{do}$ not exhibit a common directionality pattern. Overall the anticipatory component appears to be more salient for TDY than for JY in /VIV/ sequences when /i/ is involved.

\section{Discussion}

In agreement with predictions from the DAC model, directionality patterns in JY coarticulation were found to depend on specific articulatory requirements for the production of consonants. Dorsals $/ \mathrm{n} /$ and $/ \mathrm{k} /$ in all sequences and the lingual fricatives $/ \mathrm{s} /$ and $/ \mathrm{S} /$ in sequences without /i/ exhibit similar trends in coarticulatory direction for TDY and JY, and high TDY-JY correlations in coarticulatory duration. Indeed, $/ \mathrm{n} /$ favors the carryover component and $/ \mathrm{s} /, / \mathrm{S} /$ and $/ \mathrm{k} /$ the anticipatory component, while both prevalent coarticulatory 
directions yield specially long V-to-V effects in most cases. Coincident trends in coarticulatory direction for tongue dorsum and jaw along the vertical dimension in VCV sequences with dorsal consonants may result from the strong muscle linkages between these two articulatory structures: the jaw assists the tongue dorsum during the production of dorsal consonants, and overshoot in JY activity may take place in VCV sequences with two or three dorsal segmental units. Manner requirements may help explain why lingual fricatives favor anticipation for both TDY and JY in sequences without /i/. Prevalence of anticipation over carryover for velars may be related to forward tongue dorsum motion during the velar closure period causing prominent $\mathrm{C}$-to- $\mathrm{V}$ anticipation to occur.

This cooperative action between the jaw and the tongue dorsum was found to be less effective for consonants produced with the tongue front and a lower jaw. Indeed, $/ 1 / \mathrm{and} / \mathrm{n} /$ show differences in coarticulatory direction and duration between TDY and JY in sequences with /i/, namely, longer anticipatory effects and shorter carryover effects for TDY than for JY.

In Recasens (2002) vocalic effects were found to be generally longer in the context of fixed back /a/, /u/ vs front /i/ (TDX, TDY) and of fixed low back /a/ vs high back /u/ (TDX, TDY, TTX). However, articulatory overshoot may cause dorsal consonants to exhibit long tongue dorsum effects to occur during fixed /i/. In the present study, JY effects were also found to be longer during fixed /i/ than during fixed /a/ not only in VCV sequences with dorsal consonants (and longer during fixed /a/ than during fixed $/ \mathrm{u} /$, as expected). This finding could be associated with the relatively slow motion of the mandible in speech.

Data presented in this paper indicate that coarticulatory effects for different articulators may be strongly related to patterns of interarticulatory coordination. Thus, the jaw and the tongue dorsum may show an analogous coarticulatory behavior for consonants exhibiting a close interaction between the two articulatory structures (e.g., dorsals) but a different coarticulatory behavior for those other consonants for the production of which the tongue dorsum and the jaw do not act coordinatively (e.g., dentoalveolars). To a large extent, certain coarticulation aspects (e.g., prevalence of carryover over anticipation for V-to- $\mathrm{V}$ effects on fixed /i/) are jaw specific and may be associated with the massive structure of the mandible in speech.

In contrast with F1 coarticulation data (Recasens and Pallarès, 2001), V-to-V effects in jaw vertical displacement were not always found to be longer at the carryover vs anticipatory level in fixed /a/ condition. Indeed, mandibular effects for fricatives and, to some extent, for $/ \mathrm{p} /$ and $/ \mathrm{l} /$ on $/ \mathrm{a} /$ were reported to favor the anticipatory vs carryover component. Regarding the fixed /i/ condition, the JY data are in accordance with the F1 data in favoring anticipation for $/ \mathrm{s} /$ and $/ \mathrm{S} /$ but in disagreement with them in not favoring the carryover direction for $/ \mathrm{l} /$ and velars. Other dorsals, i.e., alveolopalatals, appear to favor the carryover component however. Differences between the results reported in our F1 study and in the present JY investigation could be partly due to the fact that isolated VCV sequences were used in the former vs the latter. They may also reflect the possibility that F1 coarticulatory effects are related to other articulatory factors besides jaw vertical displacement.

\section{Acknowledgments}

This work was supported by projects DGES PB96-0103 and BFF2000-0075-C02-01 of the Ministry of Education and Science of Spain and by research group 2001 SGR00425 of the Government of Catalonia. I would like to thank Maria Dolors Pallarès for help with the statistical analysis. 


\section{References}

Fletcher, J. and Harrington, J. (1999) Lip and jaw coarticulation, in Coarticulation. Theory, data and techniques, edited by W.J. Hardcastle and N. Hewlett (Cambridge University Press, Cambridge), pp. 164-178.

Keating, P.A., Lindblom, B., Lubker, J., and Kreiman, J. (1994) Variability in jaw height for segments in English and Swedish VCVs, Journal of Phonetics, 22, 407-422.

Magen, H.S. (1997) The extent of vowel-to-vowel coarticulation in English, Journal of Phonetics, 25, 187-206.

Recasens, D. (2002) An EMA study of VCV coarticulatory direction, Journal of the Acoustical Society of America, 111.

Recasens, D. and Pallarès, M.D. (2001) Coarticulation, blending and assimilation in Catalan consonant clusters, Journal of Phonetics, 29, 273-301.

Recasens, D. and Pallarès, M.D. (2000) A study of F1 coarticulation in VCV sequences, Journal of Speech and Hearing Research, 43, 501-512.

Recasens, D., Pallarès, M.D. and Fontdevila, J. (1997) A model of lingual coarticulation based on articulatory constraints, Journal of the Acoustical Society of America, 102, 544-561.

Sussman, H.M., MacNeilage, P.F., and Hanson, R.J. (1973) Labial and mandibular dynamics during the production of bilabial consonants: Preliminary observations, Journal of the Acoustical Sociey of America, 16, 397-420.

Tuller, E., Harris, K.S., and Gross, R. (1981) Electromyographic study of the jaw muscles during speech, Journal of Phonetics, 9, 175-188. 